\title{
Comparative Study of Purine and Pyrimidine Nucleoside Analogues Acting on the Thymidylate Kinases of Mycobacterium tuberculosis and of Humans
}

\author{
Sylvie Pochet, ${ }^{[b]}$ Laurence Dugué, $^{[b]}$ Gilles Labesse, ${ }^{[c]}$ Muriel Delepierre, ${ }^{[d]}$ and \\ Hélène Munier-Lehmann*[a]
}

Thymidine monophosphate kinase (TMPK) from Mycobacterium tuberculosis (TMPKmt) is an attractive target for the design of specific inhibitors. This fact is the result of its key role in the thymidine pathway and of unique structural features in the active site observed by X-ray crystallography, especially in comparison to its human counterpart (TMPKh). Different 5-modified thymidine derivatives, as well as purine and pyrimidine analogues or

\section{Introduction}

Nucleoside analogues such as zidovudine and stavudine, or acyclovir and ganciclovir, have demonstrated strong antiviral properties and are successfully used in the treatment of HIV or herpes virus infections. ${ }^{[1]}$ Administered as prodrugs, these compounds are phosphorylated by cellular or viral kinases, and the resulting nucleoside triphosphates have been shown to inhibit viral DNA synthesis by different mechanisms. ${ }^{[2]}$ The enzymes involved in the first two steps of activation, thymidine kinase (TK) and thymidine monophosphate kinase (TMPK), are essential members of the thymine metabolic pathway. Similarly, deoxycytidine analogues, ${ }^{[3]}$ such as zalcitabine and gemcitabine, are phosphorylated by deoxycytidine kinase and cytidine monophosphate kinase. Characterization of the enzymes involved in these phosphorylation steps is a prerequisite for the design of new specific prodrugs or inhibitors.

In the case of anti-herpes-virus agents, the most frequently used compounds are derivatives of guanine (acyclovir, ganciclovir) or 5-substituted pyrimidines (brivudin, idoxuridine, trifluridine). ${ }^{[1]}$ To be active on the viral DNA polymerases, these compounds need to be phosphorylated to their triphosphate forms. The first phosphorylation step is achieved by a virusencoded protein (HSV or VZV TK or CMV protein kinase) and is therefore restricted to infected cells. After conversion of these analogues into the corresponding monophosphates, they are further phosphorylated to nucleoside diphosphates, by TK in the case of the thymine derivatives or by cellular kinases in the other cases. The crystal structure of HSV-1 TK ${ }^{[4]}$ reveals the presence of a fold common with the nucleoside monophosphate kinase (NMPK) family, made up of a five-stranded parallel $\beta$ sheet and
C-nucleosides were tested on TMPKmt and TMPKh, and the results were rationalized by docking studies. 5-Halogenated 2'-deoxyuridines are the best inhibitors of TMPKmt found and present the highest selectivity indexes in favor of TMPKmt.

\section{KEYWORDS:}

inhibitors $\cdot$ kinases $\cdot$ purine $\cdot$ pyrimidine $\cdot$ nucleosides other additional structural elements. This fold, part of the protein core, contains the active site. Moreover, an insertion loop of HSV1 TK is analogous to the LID domain of NMPKs, which covers the active site upon binding of adenosine triphosphate (ATP). HSV1 TK and TMPKs exhibit an overall sequence identity below $16 \%$. However, crystal structures of binary complexes of $\mathrm{TK}^{[5,6]}$ or TMPKs $^{[7-10]}$ revealed a similar orientation of the enzyme-bound substrate relative to the catalytic centre. The position of the substrate appeared shifted by about $2 \AA$ after structure superimposition based on the strictly conserved residues.

[a] Dr. H. Munier-Lehmann

Institut Pasteur

Laboratoire de Chimie Structurale

des Macromolécules (URA CNRS 2185)

28, Rue du Dr Roux, 75724 Paris Cedex 15 (France)

Fax: (+ 33) 14061-3963

E-mail:hmunier@pasteur.fr

[b] Dr. S. Pochet, L. Dugué

Institut Pasteur

Unité de Chimie Organique (URA CNRS 2128)

28, Rue du Dr Roux, 75724 Paris Cedex 15 (France)

[c] Dr. G. Labesse

Centre de Biochimie Structurale (UMR 5048)

Faculté de Pharmacie

Université de Montpellier I

34000 Montpellier (France)

[d] Dr. M. Delepierre Institut Pasteur

Unité de Résonance Magnétique Nucléaire

des Biomolécules (URA CNRS 2185)

28, Rue du Dr Roux, 75724 Paris Cedex 15 (France) 
The recent characterization of TMPK from Mycobacterium tuberculosis (TMPKmt) in our laboratory revealed new structural and catalytic features, which make the enzyme a favored target for antituberculosis drugs. ${ }^{[11]}$ Some thymidine-5'-O-monophosphate analogues were shown to be inhibitors of TMPKmt. ${ }^{[11-13]}$ For therapeutic application, these analogues should be administered as nucleosides, which should be phosphorylated to their nucleotide monophosphate counterparts by an M.tuberculosis protein. However, there is no TK activity in this bacterium, ${ }^{[14,15]}$ which seemingly renders the use of TMPKmt as a target invalid. Our findings that 5-bromo- 2 '-deoxyuridine (5BrdU) and 3 '-azido$3^{\prime}$-deoxythymidine (AZT) are inhibitors of TMPKmt as potent as their nonphosphorylated $5^{\prime}$-modified derivatives remove this barrier. ${ }^{[16]}$ No metabolic processing would be necessary for these compounds to become pharmacologically active, which opens new avenues in the search for specific inhibitors of TMPKmt as antituberculosis drugs. Unlike in the treatment of anti-herpesvirus infections, these compounds would directly act on their target, TMPKmt.

In the study reported herein, the thymine moiety of thymidine (dT) was explored in particular. Different purine and pyrimidine nucleoside analogues, as well as C-nucleosides known to be more stable in vivo and nonheterocyclic nucleosides, were tested as inhibitors of purified TMPKmt. Some antiherpetic inhibitors were selected, as their anabolism and catabolism have been extensively studied. We also checked for specificity by carrying out parallel tests on human TMPK (TMPKh) and the experimental data were rationalized by docking studies.

\section{Results and Discussion}

The inhibitory potency of base-modified dT analogues was tested on recombinant TPMKh, purified as described in the Experimental Section, and on TMPKmt as described previously. ${ }^{[1]}$ From the known crystal structure of TMPKmt complexed with dTMP (Protein Databank (pdb) accession number: 1G3U) ${ }_{1}^{[10]}$ the potential effect of chemical substitutions was analyzed. A comparative analysis of the crystal structures of TMPKmt and TMPKh (pdb accession number: PDB1E2Q) ${ }^{[17]}$ was also performed, to assess the selectivity of the explored compounds on the two TMPKs.

\section{The 5-position of the pyrimidine ring}

Substitution of the 5-methyl group with a halogen atom or an alkyl chain was explored first (Table 1). The 5-halogenated deoxyuridines exhibited affinities for TMPKmt similar to those of their corresponding nucleoside monophosphates, which are substrates of this enzyme. ${ }^{[11]}$ However, these compounds are much less potent towards TMPKh in comparison to their nucleotide counterparts: the Michaelis constant $K_{\mathrm{m}}$ for dTMP $(5 \mu \mathrm{M})$ is 40 times lower than the inhibition constant $K_{\mathrm{i}}$ for dT $(180 \mu \mathrm{M})$. Despite significant structural similarities, TMPKmt and TMPKh show only $23 \%$ sequence identity. Analysis of the active site entrance in the vicinity of the 5 -position of the ribose moiety highlighted significant amino acid differences (Figure 1). We predicted that D163 in TMPKmt should be
Table 1. Evaluation of the inhibitory potencies of 5-modified dU and AZdU analogues. $\left[{ }^{[a]}\right.$

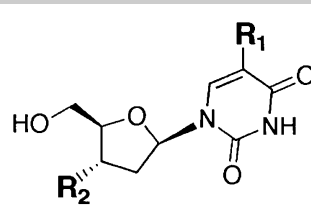

\begin{tabular}{|c|c|c|c|c|c|c|}
\hline $\mathrm{R}_{2}$ & & $\mathrm{OH}$ & & & $\mathrm{N}_{3}$ & \\
\hline $\mathrm{R}_{1}$ & Acronym & TMPKmt & TMPKh & Acronym & TMPKmt & TMPKh \\
\hline $\mathrm{CH}_{3}$ & dT & 27 & 180 & AZT & 28 & 450 \\
\hline $\mathrm{H}$ & $d U$ & 1020 & 2550 & AZdU & 810 & N.I. ${ }^{[c]}$ \\
\hline $\mathrm{F}$ & $5 F d U$ & 212 & N.I. ${ }^{[d]}$ & & & \\
\hline $\mathrm{Cl}$ & $5 \mathrm{CldU}$ & 10 & 375 & AZCldU & 16 & N.I. ${ }^{[b]}$ \\
\hline $\mathrm{Br}$ & $5 \mathrm{BrdU}$ & 5 & 214 & AZBrdU & 10.5 & N.I. ${ }^{[b]}$ \\
\hline & $5 \mathrm{ddU}$ & 33 & 350 & & & \\
\hline $\mathrm{CF}_{3}$ & $5 \mathrm{CF}_{3} \mathrm{dU}$ & 97 & 1020 & & & \\
\hline $\mathrm{OH}$ & $5 \mathrm{HOdU}$ & 270 & N.I. ${ }^{[b]}$ & & & \\
\hline $\mathrm{CH}_{2} \mathrm{OH}$ & 5HOMedU & 820 & 2700 & & & \\
\hline $\mathrm{CH}=\mathrm{CHBr}(E)$ & BVDU & 625 & 1100 & & & \\
\hline $\mathrm{CH}_{2} \mathrm{CH}_{3}$ & 5EtdU & 1140 & N.I. ${ }^{[d]}$ & & & \\
\hline
\end{tabular}

[a] Enzymatic assay conditions were as described in the Experimental Section and the figures given correspond to the $K_{\mathrm{i}}$ values in $\mu$ m. N.I., no inhibition detected at a final concentration of [b] $1 \mathrm{mmM},[\mathrm{c}] 3 \mathrm{~mm}$, and [d] $4 \mathrm{~mm}$.

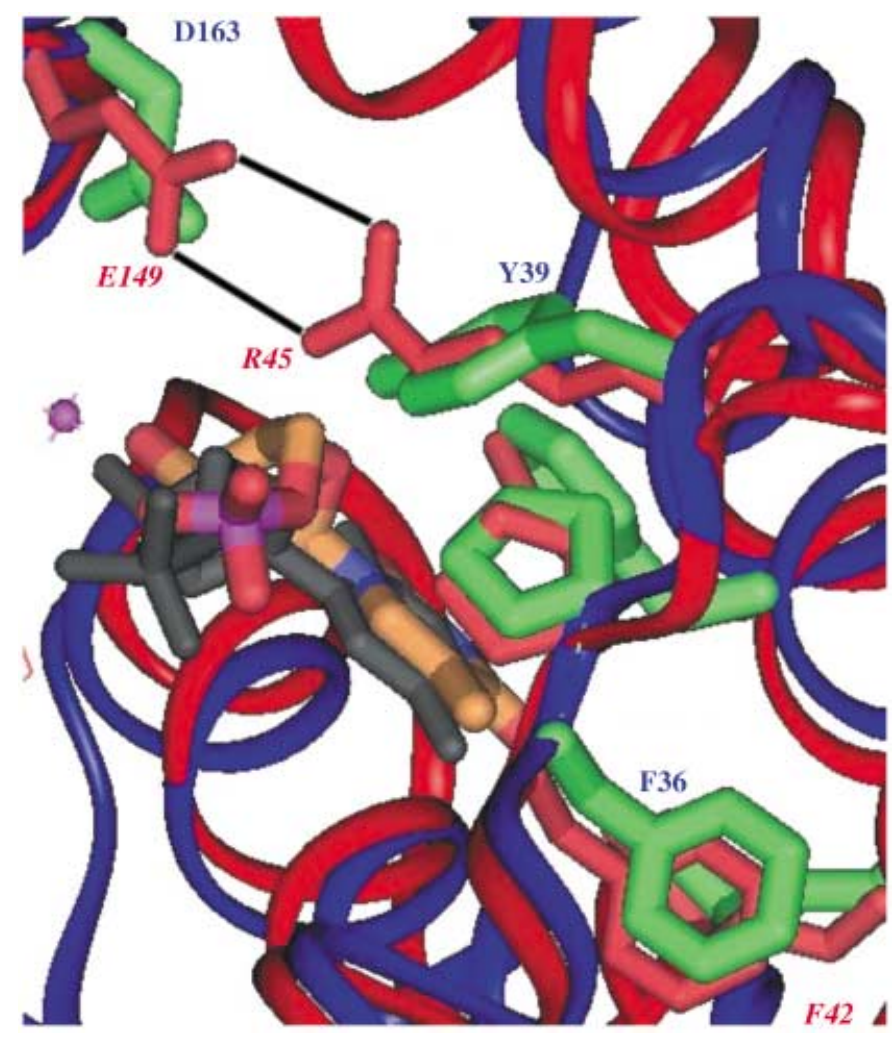

Figure 1. Active site entrance of TMPKh and TMPKmt. TMPKmt (blue ribbon) and TMPKh (red ribbon) are superposed. Side chains of F36, P37, Y39, and D163 of TMPKmt are labeled, as are the residues at similar positions in TMPKh, while the salt bridge between R45 and E149, only observed in TMPKh, is marked by black lines. dTMP is in black for TMPKh and by atom type in TMPKmt as cocrystallized. 
involved in hydrogen bonding to the 5'-hydroxy group of nucleosides. ${ }^{[16]}$ In TMPKh, this position is occupied by E149, which forms a salt bridge with R45. This latter residue is equivalent to Y39 in TMPKmt, which is connected to D163 through a bridging water molecule (W43). We propose that the 5 '-hydroxy group moves to a position mimicking that of the bridging water molecule in the TMPKmt/dTMP crystal. In TMPKh, such a rearrangement would not be favorable because of the salt bridge (R45-E149), which leads to an affinity of a nucleoside for TMPKh lower than that of the corresponding nucleotide. Consequently, the selectivity of nucleosides is in favor of TMPKmt, with selectivity indexes $\left(\mathrm{SI}=K_{\mathrm{i}} \mathrm{TMPKmt} / K_{\mathrm{i}} \mathrm{TMPKh}\right)$ between 2 (for 5-bromovinyl-2'-deoxyuridine (BVDU)) and 40 (for 5-chloro- 2 -deoxyuridine (5CldU) and $5 \mathrm{BrdU}$ ).

The presence of a chlorine or a bromine atom at the 5-position of dU molecule increases its affinity, while that of an iodine atom (bigger than a methyl group) does not. The presence of a fluorine atom (smaller than a methyl group) results in a decreased affinity of the same order as produced by the hydroxy group. The most detrimental effect was observed on the substitution of the 5-methyl group with a hydrogen atom (dU). The same tendency was observed in the $3^{\prime}$-azido series, when 5-ClAZdU and 5-BrAZdU were compared to AZdU and AZT. However, in contrast to TMPKmt, in which the $3^{\prime}$-azido group confers an affinity better than or similar to that of the $3^{\prime}$-hydroxyl counterpart, AZT exhibits a $K_{\mathrm{i}}$ value for TMPKh 2.5 times higher than that of $\mathrm{dT}$. The introduction of a bulky alkyl group other than methyl at the 5-position $\left(\mathrm{CF}_{3}, \mathrm{CH}_{2} \mathrm{OH}, \mathrm{CH}=\mathrm{CHBr}\right)$ also resulted in decreased affinity. Repulsion was expected from the substitutions of the methyl group by larger groups as a result of the numerous and close contacts observed in the dTMP/TMPKmt complex (Figure 2). The improved affinity for TMPKmt observed

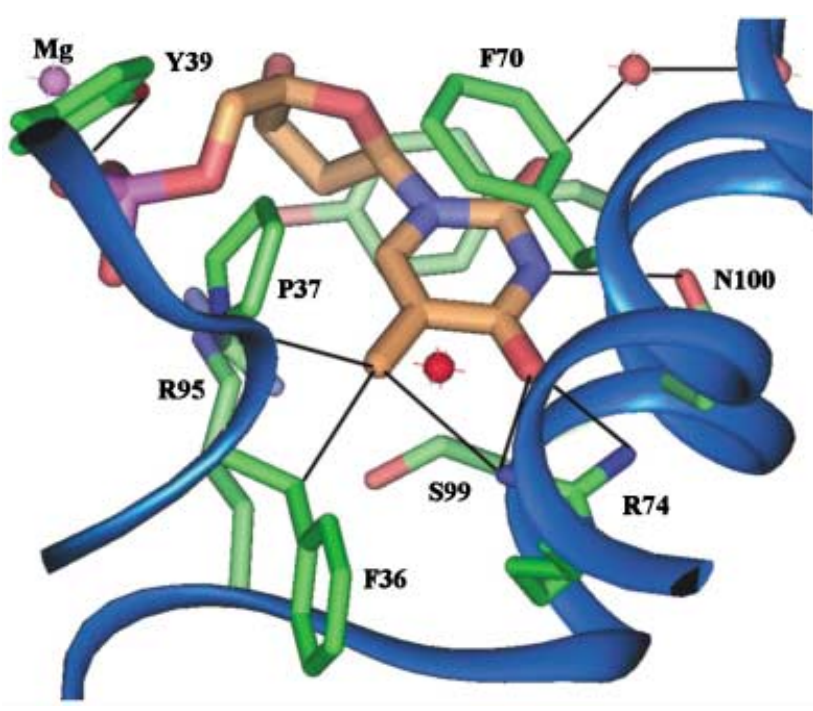

Figure 2. Orientation of dTMP in the active site pocket of TMPKmt. View from the 5-methyl group, showing the neighboring side chains of residues F36 and R74 and the main chain atom of P37. Van der Waals contacts involving the methyl group and hydrogen bonds with the nucleotide base atoms are represented as black lines, and the protein backbone as blue ribbon. Side chains of interacting residues are labeled, and the three water molecules involved in substrate binding are shown. with chloro and bromo derivatives is linked to the presence of the positively charged residue (R74) in the vicinity of the methyl binding pocket, otherwise made up only of hydrophobic side chains (F36, P37). While similar residues are observed at equivalent positions in TMPKh (R76, F42, and P43 respectively), some variability is observed in the second shell of residues surrounding the previously mentioned amino acids (e.g.: R45, N75, and D121 in TMPKh instead of Y39, D73, and E124 in TMPKmt). These latter residues do not interact directly with the substrate but rather stabilize the side chains of amino acids directly involved in base recognition. The substitution of second shell residues might affect the flexibility of the substrate binding pocket and/or the environment characteristics (hydrophobicity or dielectric constant). These substitutions might explain the distinct ordering of the 5-substituted analogues according to their affinity for TMPKh compared to that for TMPKmt. Moreover, these results suggest only little rearrangement in the vicinity of the 5-methyl group upon binding to TMPKmt. Only isosteric and isoelectronic substituents $(\mathrm{Cl}$ or $\mathrm{Br})$ with respect to the methyl group yielded inhibitors active below $10 \mu \mathrm{m}$. However a remarkable property of these inhibitors resides in their relative specificity, with the highest SI (around 40) in favor of TMPKmt.

\section{Nature of the heterocyclic moiety}

While the phosphate group was not essential for binding of dT analogues to TMPKmt, comparison with enterobacterial orthologues suggested that the aromaticity of the base moiety was important in the case of TMPKmt because of ring stacking with F70. ${ }^{[16]}$ In TMPKh, the equivalent F72 is also believed to stabilize the planar and aromatic base moiety. As expected, a decrease in or the loss of the aromaticity of the base moiety results in lower affinity for TMPKmt: dhdT and dhdU are both less effective than dT (Table 2).

Purine and pyrimidine nucleosides were also tested on TMPKmt and TMPKh (Table 2). dC and 5MedC bind poorly to TMPKmt; the presence of a positively charged amino group at C4 instead of a carbonyl group appears to be detrimental. This effect is less important in the case of TMPKh, which has a better affinity for $5 \mathrm{MedC}(\mathrm{SI}=0.5)$, despite little structure change in the vicinity of the charged amino group (see above), and might be further enhanced in TMPKmt by the accompanying rearrangement of the neighboring N100, which is hydrogen-bonded to the hydrogen-bearing nitrogen N3 of dT. Again, the presence of the 5-methyl group seems to improve the inhibitory effect. Surprisingly the purine nucleosides $d G$ and $d A$ were equally effective inhibitors of TMPKmt, with $K_{\mathrm{i}}$ values 2.5-4.7 times lower than those of cytidine analogues (5MedC or $\mathrm{dC}$, respectively). On the other hand, dl inhibits TMPKmt in the same concentration ranges as 5 MedC. The inhibition of TMPKmt by dG was further characterized and shown to be noncompetitive with respect to ATP and competitive with respect to dTMP (data not shown), which indicates a specific interaction of $d G$ with the dTMP binding site. As already reported for other TMPKs, ${ }^{[18]}$ dGMP was not a substrate for TMPKmt but was a competitive inhibitor with a $K_{\mathrm{i}}$ value of $117 \mu \mathrm{m}$. Other guanosine analogues modified in 


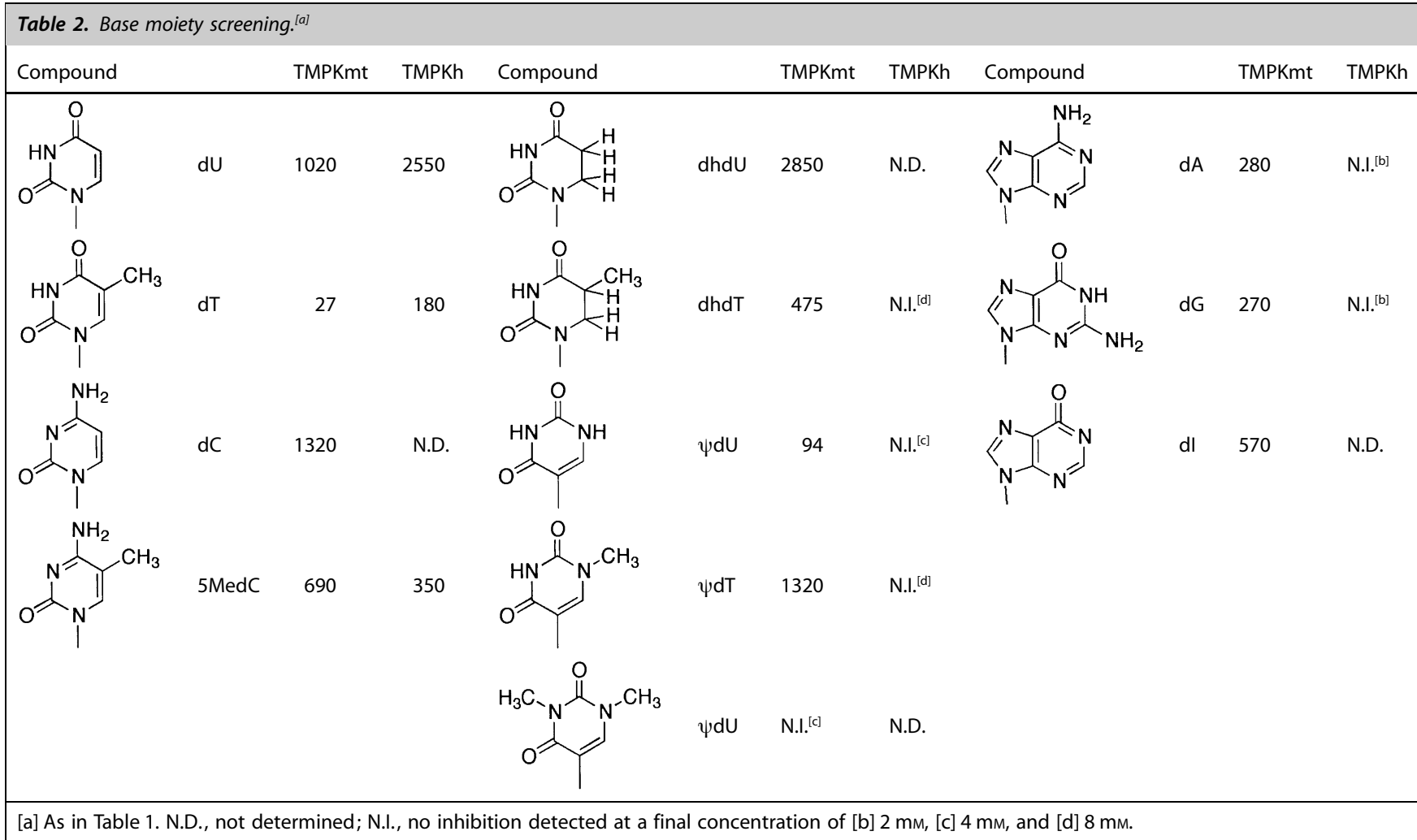

the sugar part of the molecule were tested on TMPKmt: acyclovir (ACV) and ganciclovir (GCV) are less potent than dG (by factors of 2 and 4, respectively). Positioning of the purine analogues in TMPKmt was based on conformations observed in their respective complexes with HSV-1 TK, with the ribose moiety as a main anchor. The impact of ribose moiety modification was analyzed independently by evaluation of the potential interaction changes. Little rearrangement was required for the binding to TMPKmt of acyclic polyols instead of ribose, while numerous interactions were lost, in agreement with the decreased affinity. In contrast, accommodation of the bulky purine base moieties implies significant rearrangements of the substrate-binding pocket, in particular reorientation of the N100 side chain. Favorable interactions might compensate for the cost of these conformational changes as well as discrimination between $\mathrm{dl}$ and $\mathrm{dG}$ (putative hydrogen bonding involving the amino group N4 and the hydroxy group of S103).

Three C-nucleosides ( $\psi \mathrm{dU}, \psi \mathrm{dT}, \mathrm{dm} \psi \mathrm{dU}$ ) related to $\mathrm{dT}$ and $\mathrm{dU}$ were selected, as these compounds are more stable in vivo than their corresponding $\mathrm{N}$-nucleotides. The carbon-carbon bond between the base and the sugar moiety has an increased stability over the glycosidic carbon-nitrogen bond. Consequently, C-nucleosides are not degraded by nucleosidases. Inhibitory data for these molecules are given in Table 2. $\mathrm{dm} \psi \mathrm{dU}$ exhibits a $K_{\mathrm{i}}$ value of over $5 \mathrm{~mm}$, which might be explained by van der Waals clashes between one of the two methyl groups and the N100 amide group. $\psi \mathrm{dU}$ and $\psi \mathrm{dT}$ are known to be isosters of $\mathrm{dU}$ and $\mathrm{dT}$, respectively, ${ }^{[19]}$ with identical $\mathrm{p} K_{\mathrm{a}}$ values. Surprisingly, $\psi \mathrm{dT}$ exhibits a poorer affinity for TMPKmt than $\psi d \mathrm{dU}$. Previously, the presence of a methyl group or an isosteric and isoelectronic substituent at the 5-position had yielded a better affinity, as in the case of dT versus dU or of dhdT versus dhdU. A conformational analysis in terms of sugar puckering (N/S population) and position of the base relative to the sugar (syn/anti orientation) was therefore conducted for $\psi \mathrm{dU}$ and $\psi \mathrm{dT}$. The structural analysis, by high-resolution proton NMR spectroscopy, was based on evaluation of interproton distances and analysis of coupling constants. Interproton distances were evaluated by offresonance ROESY experiments to avoid Hartmann - Hahn artefacts. ${ }^{[20]}$ To evaluate the percentage of $\mathrm{C2}^{\prime}$ endo (S) and $\mathrm{C}^{\prime}$ endo (N) conformers, a two-state analysis of sugar proton coupling constants, taking coupling constant sums into account, was conducted as described by Rinkel et al. ${ }^{[21]}$ Both for $\psi \mathrm{dU}$ and for $\psi \mathrm{dT}$, this leads to about $70-80 \%$ of $\mathrm{C}^{\prime}$ endo ( $\mathrm{S}$ sugar) conformers with a phase angle around $140^{\circ}$ (anti conformers). Although the population of the S conformer is quite high, it is in the expected range for deprotonated C-nucleosides. ${ }^{[22]}$ The similar conformations of $\psi \mathrm{dU}$ and $\psi \mathrm{dT}$ observed in solution did not shed more light on their respective inhibitory potencies. An induced-fit rearrangement of the more flexible C-nucleosides or of some protein side chains might therefore be involved. Indeed, $\psi \mathrm{dU}$ is the only compound so far tested on TMPKmt that is capable of interacting suitably with R74 and N100 in both anti and syn conformations. In the syn conformation, the carbonyl oxygen atom $\mathrm{O} 2$ would point toward the 5 ' hydroxy group of the nucleoside, at hydrogen bonding distance. In $\mathrm{dC}$ and $\mathrm{dU}$, such a conformation would place a carbon atom in front of the N100 amide group. 


\section{Conclusions}

This study has established that, for different nucleosides with variations mainly on the base moiety, the best inhibitors of TMPKmt are 5-halogenated thymidine analogues. Moreover, these compounds are highly specific for TMPKmt in comparison with TMPKh. Future investigations should aim to control the fate of these analogues in cells. 5-Halogenated 2'-deoxyuridine compounds are substrates for both TK1 and TK2. ${ }^{[23]}$ To avoid the phosphorylation of these compounds to their monophosphate derivatives by cellular TKs, other modifications on the sugar moiety would be necessary. One possibility would be modification at the $5^{\prime}$-position: in this case, the compounds would no longer be substrates for TKs, with the drawback that they might become inhibitors of TKs. Another possible way would be modification at the 2'-position. It is known that $2^{\prime}$ chloro-2'-deoxyuridine is not a substrate for TK1 and TK2, nor is it an inhibitor of these enzymes. ${ }^{[24]}$

\section{Experimental Section}

Chemistry: ${ }^{1} \mathrm{H}$ and ${ }^{13} \mathrm{C}$ NMR spectra were recorded in DMSO-d6 on a Bruker $400 \mathrm{MHz}$ instrument. Exact mass measurements were performed by J. Rozenski (Rega Institute for Medicinal Research, Leuven) on a quadrupole/orthogonal-acceleration time-of-flight tandem mass spectrometer equipped with a standard electrospray ionization (ESI) interface. Samples were injected in $\mathrm{MeOH} / \mathrm{H}_{2} \mathrm{O}(1: 1)$. Thin layer chromatography (TLC) was run on Merck silica gel $\left(F_{254}\right)$. Silica gel column chromatography was carried out on Merck 9385 silica gel. Unless otherwise stated, all chemicals were reagent grade, purchased from commercial sources and used without further purification.

2'-Deoxyadenosine $(\mathrm{dA}), 2^{\prime}$-deoxycytidine $(\mathrm{dC}), 2^{\prime}$-deoxyguanosine (dG), and thymidine (dT) were purchased from Chemlmpex. 2'Deoxypseudouridine ( $\psi \mathrm{dU})$, 1,3-dimethyl-2'-deoxypseudouridine $(\mathrm{dm} \psi \mathrm{dU})$, pseudothymidine ( $\psi \mathrm{dT}), 2^{\prime}$-deoxyuridine (dU), 5,6-dihydro-2'-deoxyuridine (dhdU), 5,6-dihydrothymidine (dhdT), 5-hydroxy-2'-deoxyuridine (5hdU), 5-hydroxymethyl-2'-deoxyuridine (5hmdU), 5BrdU, and 2'-deoxyinosine (dl) were purchased from Berry \& Associates. 5CldU, 5-fluoro-2'-deoxyuridine (5FdU), 5-iodo-2'deoxyuridine (5IdU), 5-trifluoromethyl-2'-deoxyuridine $\left(5 \mathrm{CF}_{3} \mathrm{dU}\right)$, AZT, ACV, GCV, and BVDU were purchased from Sigma-Aldrich. 5-Methyl-2'-deoxycytidine (5MedC) was a gift from P. Herdewijn (Rega Institute for Medicinal Research, Leuven).

$3^{\prime}$-Azido-2', $3^{\prime}$-dideoxyuridine (AZdU) was synthesized by the methodology previously described. ${ }^{[25]} 3^{\prime}$-Azido-2',-3'-dideoxy-5-bromouridine ( $5 \mathrm{BrAZdU}$ ) was synthesized by treatment of AZdU with acetic anhydride, followed by bromination in acetic acid and subsequent removal of the acetyl group with methanolic ammonia. ${ }^{[26]} 5 \mathrm{BrAZdU}$ was isolated by chromatography on a silica gel column, followed by HPLC on a C18 reversed-phase column (10-25\% linear gradient of acetonitrile in $10 \mathrm{~mm}$ triethyl ammonium acetate (TEAA) over $20 \mathrm{~min}$ ) in $61 \%$ yield. $R_{t}=13.7 \mathrm{~min} ;{ }^{1} \mathrm{H}$ NMR (DMSO-d6): $\delta=2.32\left(\mathrm{~m}, 1 \mathrm{H} ; \mathrm{H} 2^{\prime}\right)$, $2.46\left(\mathrm{~m}, 1 \mathrm{H} ; \mathrm{H} 2^{\prime \prime}\right), 3.60\left(\mathrm{~m}, 1 \mathrm{H} ; \mathrm{H}^{\prime}\right), 3.70\left(\mathrm{~m}, 1 \mathrm{H} ; \mathrm{H}^{\prime \prime}\right), 3.85(\mathrm{~m}, 1 \mathrm{H}$; $\left.\mathrm{H}^{\prime}\right), 4.39\left(\mathrm{~m}, 1 \mathrm{H} ; \mathrm{H}^{\prime}\right), 5.36\left(\mathrm{brs}, 1 \mathrm{H} ; 5^{\prime}-\mathrm{OH}\right), 6.03(\mathrm{dd}, J=5.4,1.0 \mathrm{~Hz}$, $1 \mathrm{H} ; \mathrm{H}^{\prime}$ ), $8.30(\mathrm{~s}, 1 \mathrm{H} ; \mathrm{H6}$ ), 11.80 (brs, $1 \mathrm{H} ; \mathrm{NH}) \mathrm{ppm} ;{ }^{13} \mathrm{C}$ NMR (DMSOd6): $\delta=37.79$ (C2'), 60.08 (C3'), $61.03\left(\mathrm{C}^{\prime}\right), 85.28$, and 85.35 (C1' and C4'), 96.57 (C5), 141.04 (C6), 150.56 (C2), 160.03 (C4) ppm. HRMS (ESIMS): calcd for $\mathrm{C}_{9} \mathrm{H}_{10} \mathrm{BrN}_{5} \mathrm{O}_{4} \mathrm{Na}^{+}: 353.9814$; found: $353.9815[\mathrm{M}+\mathrm{Na}]^{+}$.
3'-Azido-2', 3'-dideoxy-5-chlorouridine (5CIAZdU) was synthesized by C-5 chlorination with an electrophilic halogen reagent and sodium azide as described for uridine or 2'-deoxyuridine. ${ }^{[27]}$ Thus, $5^{\prime}$-acetyl-3'azido-2', $3^{\prime}$-dideoxyuridine was treated with $\mathrm{N}$-chlorosuccinimide and sodium azide in 1,2-dimethoxyethane, followed by deacetylation with $0.1 \mathrm{~m}$ sodium methoxide in methanol. $5 \mathrm{ClAZdU}$ was isolated after purification by chromatography on a silica gel column followed by HPLC on a C18 reversed-phase column (10-25\% linear gradient of acetonitrile in $10 \mathrm{~mm}$ TEAA over $20 \mathrm{~min}$ ) in $68 \%$ yield. $R_{\mathrm{t}}=$ $15.5 \mathrm{~min}$; 'H NMR (DMSO-d6): $\delta=2.32\left(\mathrm{~m}, 1 \mathrm{H} ; \mathrm{Hz}{ }^{\prime}\right), 2.45(\mathrm{~m}, 1 \mathrm{H}$; $\left.\mathrm{H} 2^{\prime \prime}\right), 3.62\left(\mathrm{~m}, 1 \mathrm{H} ; \mathrm{H}^{\prime}\right), 3.70\left(\mathrm{~m}, 1 \mathrm{H} ; \mathrm{H}^{\prime \prime}\right), 3.85\left(\mathrm{~m}, 1 \mathrm{H} ; \mathrm{H} 4^{\prime}\right), 4.38(\mathrm{~m}$, $\left.1 \mathrm{H} ; \mathrm{H3}^{\prime}\right), 5.30\left(\mathrm{brs}, 1 \mathrm{H} ; 5^{\prime}-\mathrm{OH}\right), 6.03\left(\mathrm{t}, \mathrm{J}=6.0 \mathrm{~Hz}, 1 \mathrm{H} ; \mathrm{H}^{\prime}\right), 8.30(\mathrm{~s}, 1 \mathrm{H}$; H6), 11.8 (brs, $1 \mathrm{H}$; NH) ppm; ${ }^{13} \mathrm{C}$ NMR (DMSO-d6): $\delta=37.72$ (C2'), $60.11\left(\mathrm{C}^{\prime}\right), 61.06\left(\mathrm{C}^{\prime}\right), 85.24\left(\mathrm{C}^{\prime}\right.$ and $\left.\mathrm{C}^{\prime}\right), 108.03$ (C5), 138.54 (C6), 150.45 (C2), 160.03 (C4) ppm. HRMS (ESI-MS): calcd for $\mathrm{C}_{9} \mathrm{H}_{10} \mathrm{CIN}_{5} \mathrm{O}_{4} \mathrm{Na}^{+}$: 310.0315 ; found: $310.0319[\mathrm{M}+\mathrm{Na}]^{+}$.

High-resolution NMR spectroscopy: High-resolution ${ }^{1} \mathrm{H}$ NMR spectra for the conformational analysis of $\psi \mathrm{dU}$ and $\psi \mathrm{dT}$ were recorded on a Varian Inova $500 \mathrm{MHz}$ spectrometer equipped with a triple resonance, pulsed field gradient probe with an actively shielded $z$ gradient. The concentration of the samples was $15 \mathrm{~mm}$ and the $\mathrm{pH}$ value (7.6) was in the same range as in the kinetics measurements. All spectra were recorded at $30^{\circ} \mathrm{C}$ in $\mathrm{D}_{2} \mathrm{O}$. Chemical shifts are given with respect to sodium 2,2-dimethyl-2-silapentane 5-sulfonate (DSS) as internal reference. DQF-COSY, TOCSY, and off-resonance ROESY spectra were recorded with a spectral width of $4 \mathrm{kHz}$ in both dimensions, a $90^{\circ}$ pulse of $4.4 \mathrm{~ms}, 2 \mathrm{~K}(\mathrm{~F} 1) \times 2 \mathrm{~K}(\mathrm{~F} 2)$ point data sets, zero-filled to $4 \mathrm{~K}$ in the $\mathrm{F} 1$ dimension and by use of 16,8 , and 32 scans per increment, respectively. The off-resonance ROESY experiment was recorded with a $11.4 \mathrm{kHz}$ effective spin lock field generated by a series of $30^{\circ}$ pulses over $400 \mathrm{~ms}$ with a recycle delay of $5 \mathrm{~s}$. To avoid Hartmann - Hahn artefacts, ${ }^{[20]}$ the offset of the spin lock carrier was shifted by approximately $8 \mathrm{kHz}$ from the centre of the spectrum in order to create an angle of $54.7^{\circ}$ between the effective spin lock axis and the static magnetic field. These ${ }^{1} \mathrm{H}$ NMR experiments were processed by use of shifted sine-bell windows in both dimensions.

Cloning of the human tmk gene and purification of TMPKh overexpressed in Escherichia coli: The 636-bp fragment corresponding to tmk gene coding for TMPKh was amplified by $\mathrm{PCR}^{[28]}$ with CDNA from SK melanoma cells as the matrix. The two synthetic oligonucleotides used for amplification were $5^{\prime}$-ggaattccatatggcggcccggcgcggg- $3^{\prime}$ and $5^{\prime}$-ccggctcgagtcacttccatagctcccccag$3^{\prime}$. During amplification, Ndel and Xhol restriction sites (in bold letters in the oligonucleotide sequences) were created at both ends of the amplified fragment. After digestion by Ndel and Xhol, the amplified gene was inserted into the PET28a plasmid (Novagen, Inc.) digested with the same enzymes. Two clones containing the tmk gene and overexpressing TMPKh with a His-Tag at the N-terminal end were characterized. One of these clones was kept for further studies and the corresponding plasmid was named pHL50-7. The DNA insert was sequenced by the double-stranded dideoxynucleotide sequencing technique ${ }^{[29]}$ in order to verify the absence of any mutational events in the course of amplification. The BL21(DE3)/pDIA17 E. coli strain, ${ }^{[30]}$ transformed with pHL50-7 plasmid, was grown in $2 \mathrm{YT}$ medium containing chloramphenicol $\left(30 \mu \mathrm{g} \mathrm{m}^{-1}\right)$ and kanamycine $\left(70 \mu \mathrm{g} \mathrm{mL}^{-1}\right)$ until the absorbance at $600 \mathrm{~nm}, A^{600}$ reached 1.5. After induction with $1 \mathrm{~mm}$ isopropyl- $\beta$-D-thiogalactopyranoside and growth for $3 \mathrm{~h}$ at $37^{\circ} \mathrm{C}$, cells were harvested, resuspended in buffer $\mathrm{A}$ ( $50 \mathrm{~mm}$ phosphate buffer, $\mathrm{pH} 7$, containing $300 \mathrm{~mm} \mathrm{NaCl}, 5 \mathrm{~mm}$ imidazole, and protease inhibitors (Complete ethylenediaminetetraacetate-free, Roche)) and broken by sonication. After centrifugation at $14000 \mathrm{rpm}$ for $30 \mathrm{~min}$ at $4{ }^{\circ} \mathrm{C}$, the supernatant was placed on a TALON column (Clontech Laboratories, Inc.) pre-equilibrated 
with buffer A. The column was extensively washed with buffer $A$ and the protein was eluted with phosphate buffer $(50 \mathrm{~mm}, \mathrm{pH} 7)$ containing $\mathrm{NaCl}(300 \mathrm{~mm})$ and imidazole $(150 \mathrm{~mm})$. Fractions with enzymatic activity were pooled and dialysed against tris(hydroxymethyl)aminomethane (Tris) $\mathrm{HCl}(50 \mathrm{~mm}, \mathrm{pH} 7.4)$ and $20 \%$ glycerol and kept at $-20^{\circ} \mathrm{C}$.

Enzymatic assays: Activity was determined as described previously $^{[31]}$ by the coupled spectrophotometric assay at $334 \mathrm{~nm}$ in an Eppendorf ECOM 6122 photometer. The reaction medium $(0.5 \mathrm{~mL}$ final volume) contained Tris- $\mathrm{HCl}(50 \mathrm{~mm}, \mathrm{pH} 7.4), \mathrm{KCl}(50 \mathrm{~mm}), \mathrm{MgCl}_{2}$ $(2 \mathrm{~mm}), \mathrm{NADH}(0.2 \mathrm{~mm})$, phosphoenol pyruvate $(1 \mathrm{~mm})$, and lactate dehydrogenase, pyruvate kinase, and nucleoside diphosphate kinase ( 2 units each). 1 unit enzyme activity corresponds to $1 \mathrm{mmole}$ product formed in $1 \mathrm{~min}$ at $30^{\circ} \mathrm{C}$ and $\mathrm{pH}$ 7.4. The concentrations of ATP and dTMP were kept constant at $0.5 \mathrm{~mm}$ and $0.05 \mathrm{~mm}$ respectively, whereas the concentrations of analogues varied between 0.005 and $8 \mathrm{~mm}$. Equation (1) was used to calculate the $K_{i}$ values with the aid of Equations (2) and (3) (classical competitive inhibition model following the Lineweaver-Burk representation):

$$
\begin{aligned}
& K_{\mathrm{i}}=\frac{K_{\mathrm{m}}[\mathrm{l}]}{\left(\mathrm{v} / \mathrm{vi}_{\mathrm{i}}-1\right)\left(K_{\mathrm{m}}+[\mathrm{S}]\right)} \\
& v=\frac{V_{\mathrm{m}}[\mathrm{S}]}{[\mathrm{S}]+K_{\mathrm{m}}} \\
& v_{\mathrm{i}}=\frac{V_{\mathrm{m}}[\mathrm{S}]}{[\mathrm{S}]+K_{\mathrm{m}}\left(1+\left[\mathrm{li} / \mathrm{Ki}_{\mathrm{i}}\right)\right.}
\end{aligned}
$$

where $v$ and $v_{\mathrm{i}}$ are the reaction velocities in the absence and in the presence of the analogue at a concentration [I], respectively, $K_{\mathrm{m}}$ is the $K_{\mathrm{m}}$ value for dTMP (4.5 $\mu \mathrm{m}$ for TMPKmt and $5 \mu \mathrm{m}$ for TMPKh), and $[S]$ is the concentration of dTMP $(50 \mu \mathrm{M})$.

Molecular modeling and docking studies: Three-dimensional structure visualization and manual superimposition were performed with the $\mathrm{XmMol}$ software. ${ }^{[32 \mathrm{a}]}$ Structure-driven sequence alignments and sequence-dependent structure superpositions were performed by use of in-house software. ${ }^{[32 b]}$ Simple substitutions were performed on the substrate molecule in the dTMP/TMPKmt complex. ${ }^{[10]}$ The more divergent analogues (compounds with a base or a sugar moiety different from thymine or ribose, respectively), which includes those crystallized in complexation with HSV-1 TK, were docked in the crystal structure of TMPKmt by using the similarity with the original substrate. This allowed a visual inspection of the structures of the complexes and analysis of the ligand-protein contacts.

This work was supported by grants from the Institut Pasteur, the Institut National de la Santé et de la Recherche Médicale, and the Centre National de la Recherche Scientifique (Grant nos. URA 2185 and 2128, and UMR 5048) and the Ministère de la Recherche (ACI). We are grateful to J. Rozenski for the mass spectrometry experiments, to P. Herdewijn for the kind gift of 5MedC, and to O. Barzu for support and careful reading of the manuscript.
[1] E. De Clercq, J. Clin. Virol. 2001, 22, 73.

[2] E. De Clercq, Nat. Rev. Drug Discovery 2002, 1, 13.

[3] T. Obata, Y. Endo, D. Murata, K. Sakamoto, T. Sasaki, Curr. Drug Targets 2003, 4, 305.

[4] D. G. Brown, R. Visse, G. Sandhu, A. Davies, P. J. Rizkallah, C. Melitz, W. C. Summers, M. R. Sanderson, Nat. Struct. Biol. 1995, 2, 876.

[5] J. N. Champness, M.S. Bennett, F. Wien, R. Visse, W. C. Summers, P. Herdewijn, E. de Clercq, T. Ostrowski, R. L. Jarvest, M. R. Sanderson, Proteins 1998, 32, 350.

[6] C. Wurth, U. Kessler, J. Vogt, G. E. Schulz, G. Folkers, L. Scapozza, Protein Sci. 2001, 10, 63.

[7] A. Lavie, M. Konrad, R. Brundiers, R. S. Goody, I. Schlichting, J. Reinstein, Biochemistry 1998, 37, 3677.

[8] A. Lavie, N. Ostermann, R. Brundiers, R. S. Goody, J. Reinstein, M. Konrad, I. Schlichting, Proc. Natl. Acad. Sci. U.S.A. 1998, 95, 14045.

[9] N. Ostermann, I. Schlichting, R. Brundiers, M. Konrad, J. Reinstein, T. Veit, R. S. Goody, A. Lavie, Struct. Fold. Des. 2000, 8, 629.

[10] I. Li de la Sierra, H. Munier-Lehmann, A. M. Gilles, O. Barzu, M. Delarue, J. Mol. Biol. 2001, 311, 87.

[11] H. Munier-Lehmann, A. Chaffotte, S. Pochet, G. Labesse, Protein Sci. 2001, 10, 1195.

[12] V. Vanheusden, H. Munier-Lehmann, S. Pochet, P. Herdewijn, S. Van Calenbergh, Bioorg. Med. Chem. Lett. 2002, 12, 2695.

[13] A. Haouz, V. Vanheusden, H. Munier-Lehmann, M. Froeyen, P. Herdewijn, S. Van Calenbergh, M. Delarue, J. Biol. Chem. 2003, 278, 4963.

[14] H. Saito, H. Tomioka, J. Gen. Microbiol. 1984, 130, 1863.

[15] S. T. Cole, R. Brosch, J. Parkhill, T. Garnier, C. Churcher, D. Harris, S. V. Gordon, K. Eiglmeier, S. Gas, C. E. Barry 3rd, F. Tekaia, K. Badcock, D. Basham, D. Brown, T. Chillingworth, R. Connor, R. Davies, K. Devlin, T. Feltwell, S. Gentles, N. Hamlin, S. Holroyd, T. Hornsby, K. Jagels, B. G. Barrell, Nature 1998, 393, 537.

[16] S. Pochet, L. Dugué, D. Douguet, G. Labesse, H. Munier-Lehmann, ChemBioChem 2002, 3, 108.

[17] N. Ostermann, A. Lavie, S. Padiyar, R. Brundiers, T. Veit, J. Reinstein, R. S. Goody, M. Konrad, I. Schlichting, J. Mol. Biol. 2000, 304, 43.

[18] A. Y. S. Jong, J. L. Campbell, J. Biol. Chem. 1984, 259, 14394

[19] B. K. Bhattacharya, R. V. Devivar, G. R. Revankar, Nucleosides Nucleotides $1995,14,1269$.

[20] H. Desvaux, P. Berthault, N. Birlirakis, M. Goldman, J. Magn. Reson. 1994, 108, 219.

[21] L. J. Rinkel, C. Altona, J. Biomol. Struct. Dyn. 1987, 4, 621.

[22] I. Luyten, C. Thibaudeau, A. Sandstrom, J. Chattopadhyaya, Tetrahedron $1997,53,6433$.

[23] S. Eriksson, B. Kierdaszuk, B. Munchpetersen, B. Oberg, N. G. Johansson, Biochem. Biophys. Res. Commun. 1991, 176, 586.

[24] B. Kierdaszuk, K. Krawiec, Z. Kazimierczuk, U. Jacobsson, N. G. Johansson, B. Munch-Petersen, S. Eriksson, D. Shugar, Nucleosides Nucleotides 1999, $18,1883$.

[25] T.-S. Lin, W. R. Mancini, J. Med. Chem. 1983, 26, 544.

[26] T.-S. Lin, M. S. Chen, C. McLaren, Y.-S. Gao, I. Ghazzouli, W. H. Prusoff, J. Med. Chem. 1987, 30, 440

[27] R. Kumar, L. I. Wiebe, E. E. Knaus, Can. J. Chem. 1994, 72.

[28] J. Sambrook, E. F. Fritsch, T. Maniatis, Molecular Cloning, a Laboratory Manual (2nd Edition), Cold Spring Harbor Laboratory Press, 1989.

[29] F. Sanger, S. Nicklen, A. R. Coulson, Proc. Natl. Acad. Sci. U.S.A. 1977, 74, 5463.

[30] H. Munier, A.-M. Gilles, P. Glaser, E. Krin, A. Danchin, R. S. Sarfati, O. Bârzu, Eur. J. Biochem. 1991, 196, 469.

[31] C. Blondin, L. Serina, L. Wiesmüller, A.-M. Gilles, O. Bârzu, Anal. Biochem. 1994, 220, 219

[32] a) P. Tuffery, J. Mol. Graphics 1995, 13, 67; b) V. Catherinot and G. Labesse, unpublished work.

Received: April 1, 2003 [F 608] 